\title{
Invasive pulmonary aspergillosis in immunocompetent patients hospitalised with influenza A-related pneumonia: a multicenter retrospective study
}

\author{
Liang Chen ${ }^{1 *} \mathbb{D}$, Xiudi Han ${ }^{2}$, Yanli Li $i^{3}$, Chunxiao Zhang ${ }^{4}$ and Xiqian Xing ${ }^{5}$
}

\begin{abstract}
Background: Increasing cases of pulmonary aspergillosis (IPA) in immunocompetent patients with severe influenza have been reported. Howevere, the risk factors for occurence and death are largely unknown.

Methods: Data of hospitalised patients with influenza A-related pneumonia (FluA-p) obtained from five teaching hospitals from 2031 to 2018, were reviewed. Univariate and multivariate logistical regression analyses were performed to determine the risk factors involved in the acquisition and 60-day mortality in IPA patients.

Results: Of the 693 FluA-p patients included in the study, 3.0\% (21/693) were IPA patients with a 60-day mortality of $42.9 \%$ (9/21). Adjusted for confounders, a Cox proportional hazard model showed that IPA was associated with increased risk for 60-day mortality [hazard ratio (HR) 4.336, 95\% confidence interval (CI) 1.191-15.784, $p=0.026$ ] in FluA-p patients. A multivariate logistic regression model confirmed that age (odd ratio (OR) 1.147, 95\% Cl 1.048$1.225, p=0.003$ ), systemic corticosteroids use before IPA diagnosis (OR 33.773, 95\% Cl 5.681-76.764, $p<0.001$ ), leukocytes $>10 \times 10^{9} / \mathrm{L}(\mathrm{OR} 1.988,95 \% \mathrm{Cl} 1.028-6.454, p=0.029)$ and lymphocytes $<0.8 \times 10^{9} / \mathrm{L}$ on admission (OR $34.813,95 \% \mathrm{Cl} 1.676-73.006, p=0.022)$, were related with the acquisition of IPA. Early neuraminidase inhibitor use (OR 0.290, 95\% Cl 0.002-0.584, $p=0.021$ ) was associated with a decreased risk for a 60-day mortality in IPA patients.

Conclusions: Our results showed that IPA worsen the clinical outcomes of FluA-p patients. The risk factors for the acquisition and death were helpful for the clinicians in preventing and treating IPA.
\end{abstract}

Keywords: Influenza A, Pneumonia, Invasive pulmonary aspergillosis, Risk factor

\section{Background}

Influenza is a respiratory infectious disease, caused by influenza viruses, and which can present seasonal epidemics and pandemics $[1,2]$. Despite the progress in medical technologies and economic development, influenza still causes considerate complications and mortality [3]. Following infection by influenza viruses, patients can

\footnotetext{
* Correspondence: chenliang1995@sina.com

'Department of Infectious Diseases, Beijing Jishuitan Hospital, 4th Medical College of Peking University, Beijing, China

Full list of author information is available at the end of the article
}

show a broad spectrum of clinical symptoms, ranging from self-limited upper respiratory tract illness to severe pneumonia and acute respiratory distress syndrome (ARDS) [4]. It was estimated that every year, $10-20 \%$ of the global population experienced symptomatic influenza, including 3-5 million severe illnesses and 260-640 thousand deaths [5].

Nearly half of severe influenza patients present with pneumonia, which is mostly caused by influenza A [6]. Influenza pneumonia is often coinfected with other pathogens and this worsen the clinical symptoms and

(c) The Author(s). 2020 Open Access This article is licensed under a Creative Commons Attribution 4.0 International License, which permits use, sharing, adaptation, distribution and reproduction in any medium or format, as long as you give appropriate credit to the original author(s) and the source, provide a link to the Creative Commons licence, and indicate if changes were made. The images or other third party material in this article are included in the article's Creative Commons licence, unless indicated otherwise in a credit line to the material. If material is not included in the article's Creative Commons licence and your intended use is not permitted by statutory regulation or exceeds the permitted use, you will need to obtain permission directly from the copyright holder. To view a copy of this licence, visit http://creativecommons.org/licenses/by/4.0/ The Creative Commons Public Domain Dedication waiver (http://creativecommons.org/publicdomain/zero/1.0/) applies to the data made available in this article, unless otherwise stated in a credit line to the data. 
deteriorates the outcomes [7, 8]. Previous studies found, that Streptococcus pneumoniae, Staphylococcus aureus and Haemophilus influenzae, were the most common etiologies in coinfected influenza [9]. The Chinese and American guidelines recommend empiric antibiotics use to fight the pathogens associated with severe influenza $[10,11]$. Invasive pulmonary aspergillosis mostly and traditionally occurs in immunocompromised hosts, such as patients with hematopoietic stem cell transplantation, granulocyte deficiency and organ transplant recipients; but rarely in those with normal immune function [12, 13]. In recent years, more cases of IPA have been reported in severe influenza patients and with increased mortality [14-16]. The most notable was that over $30 \%$ of these cases had no classic immunocompromised factors.

However, there are limited data on influenzaassociated pulmonary aspergillosis (IAPA), especially in prior immunocompetent patients. The incidence and disease characteristics were largely unknown. Identifying those patients with high risk for IPA, remains challenging. We carried out this multicenter retrospective study with the following purposes: i) to investigate the incidence and risk factors for IPA acquisition in immunocompetent, adult patients hospitalised with influenza A-related pneumonia (FluA-p); ii) to explore the risk factors associated with a 60-day mortality in IAPA patients.

\section{Methodology}

\section{Study design and population}

We screened hospitalised patients for positive influenza A virus RNA using respiratory specimens by reverse transcription polymerase chain reaction (RTPCR) from microbiology laboratories of five teaching hospitals in Beijing, Shandong and Yunnan Provinces and during the period from 1st January to 31st December 2018 (the details of the five centers are shown in Additional file 1: Appendix file 1). From this data, we retrieved all cases had both influenza and radiograph proven pneumonia.

Patients were excluded if [17] (1) age < 18 years; (2) pneumonia onset $\geq 48 \mathrm{~h}$ after admission and not been hospitalised within the last 28 days, because the consensus of nosocomial pneumonia caused by influenza was difficult; (3) Immunocompromised status [18].

\section{Group division}

We divided the patients into two groups: the case group included patients with FluA-p, who subsequently became infected with proven and/or probable invasive pulmonary aspergillosis (IPA group); and the control group that included patients with FluA-p and who showed no evidence of Aspergillus infection while hospitalised.

\section{Study definitions}

Patients with FluA-p were defined as patients with respiratory symptoms and a new pulmonary infiltrate on the chest radiograph, combined with positive influenza virus A RT-PCR testing during the influenza seasons.

A Proven IPA was defined as the microscopic evidence of dichotomous branching hyphae with a positive culture for Aspergillus through an endobronchial biopsy, irrespective of host factors or clinical features [18].

According to the revised definitions of invasive fungal diseases from the European Organization for Research and Treatment of Cancer/Invasive Fungal Infections Cooperative Group and the National Institute of Allergy and Infectious Diseases Mycoses Study Group (EORTC/ MSG) in 2019 [18], a probable IPA required a host factor, clinical features and mycological evidence of aspergillosis. However, these criteria were created for immunosuppressed hosts and influenza-related aspergillosis may occur in previously normal hosts. Thus, the host factors were not required in our study. The clinical features included one of the following signs or symptoms: refractory fever to at least 3 days of appropriate antibiotic treatment; recrudescent fever after a period of defervescence of at least $48 \mathrm{~h}$, while still on antibiotics and without other apparent cause; dyspnea; hemoptysis; pleural friction rub or chest pain; worsening respiratory insufficiency in spite of appropriate antibiotic therapy and ventilatory support. The radiological criteria included any infiltrates on pulmonary imaging detected by chest $\mathrm{x}$-ray or CT scan of the lungs. The mycological evidence included: a positive Aspergillus culture from a bronchoalveolar lavage (BAL); a galactomannan (GM) optical index on BAL of $\geq 1.0$; a GM optical index on serum of $\geq 0.5$ [18].

Early neuraminidase inhibitor (NAI) treatment was defined as any NAI (e.g., oseltamivir, zanamivir and peramivir) administered within 2 days after disease onset [10,11]. The coinfection with other Aspergillus pathogens was defined by community-acquired respiratory co-pathogens that was identified within 2 days of hospital admission [19]. The conditions of a community-acquired respiratory co-pathogen was defined as the definite or probable etiology (Additional file 1: Appendix 2). Immunocompetent hosts were defined as patients without immunocompromised factors described above.

\section{Data collection}

Data was retrospectively collected and included demographic information, underlying disease (Additional file 1: Appendix 3), clinical manifestations, laboratory and radiological findings, microbiologic diagnosis, treatment (use of antiviral agents, corticosteroids, vasopressors and mechanical ventilation), clinical outcomes 
(complications during hospitalisation, admittance to the intensive care unit (ICU) and a 60-day mortality).

\section{Statistical analysis}

All data were analyzed with SPSS 22.0 and measurement data were tested for normality by KolmogorovSmirnov. The measurement data of normal distributions were reported as mean \pm standard deviation. Measurements data of non-normal distributions were reported as median. The categorical variables were analyzed by the Chi-square test or Fisher's exact test, and continuous variables were analyzed by the student t-test or the Mann-Whitney U-test. A $p$-value of $<0.05$ was considered statistically significant and all probabilities were two-tailed. A Cox proportional hazard model was performed to evaluate the effect of IPA on a 60-day mortality in FluA-p patients. The model was adjusted by age, gender, comorbidities, leukocytes $>10 \times 10^{9} / \mathrm{L}$, serum procalcitonin $>0.1 \mathrm{ng} /$ $\mathrm{ml}$, coinfection with non-Aspergillus pathogens and early use of neuraminidase inhibitors. The clinical characteristics were compared between patients in the IPA and control groups (Table 1). Variables with $p$ values of $\leq 0.05$ on univariate analysis were subsequently entered into the backward stepwise logistic regression analysis to identify risk factors for the acquisition and death in IPA patients.

\section{Results}

\section{Screening process}

We screened 2187 hospitalised patients with positive influenza A RNA. Overall, 693 immunocompetent adult patients hospitalised with FluA-p onset in the community were entered into the final analysis. The proportion of patients who developed IPA during hospitalisation was 3.0\% (21/693) (Fig. 1).

\section{Overview of patients with FluA-p}

Overall, the median age was 61.0 years old and the male accounted for $66.5 \%$ (461/693). Fifty-eight percent of patients $(402 / 693)$ had at least one underlying disease with the top three being diabetes mellitus 13.3\% (92/693), cerebrovascular disease $10.4 \%(72 / 693)$ and chronic obstructive pulmonary disease $5.8 \%$ (40/693). The proportion of patients with $\mathrm{PO}_{2} / \mathrm{FiO}_{2}<300 \mathrm{mmHg}$ was $53.2 \%$ (340/639). Cavities and multiple nodules in chest radiology were seen in $2.7 \%(19 / 693)$ and $21.8 \%(151 / 693)$ of patients, respectively.

Nineteen percent $(132 / 693)$ of FluA-p patients used a systemic dose of $0.6 \pm 0.3 \mathrm{mg} / \mathrm{kg}$ corticosteroids before IPA diagnosis. All patients were administrated with NAI during the disease course, while $33.3 \%$ (231/693) received NAIs within the $48 \mathrm{~h}$ after illness onset. $24.1 \%(167 / 693)$ of patients had complications with respiratory failure, $21.2 \%(147 / 693)$ had heart failure, 5.2\% (36/693) had septic shocks during hospitalisation, $26.3 \%(182 / 693)$ of patients were admitted to intensive care unit (ICU) and the 60-day mortality was $20.9 \%(145 / 693)$ (Table 1$)$.

\section{Comparisons between the IPA and control patients}

Compared with control patients, the IPA patients were older $(67.0$ yrs. vs. 60.0 yrs., $p<0.001)$, had more frequency of diabetic $(71.4 \%$ vs. $11.5 \%, p<0.001)$, chronic pulmonary disease $(28.6 \%$ vs $5.1 \%, p<0.001)$, asthma $(14.3 \%$ vs. $2.4 \%, p=0.009)$ and chronic heart failure (14.3\% vs. $0.0 \%, p<0.001)$, and lower levels of body mass index (BMI) $\left[(21.5 \pm 0.4) \mathrm{kg} / \mathrm{m}^{2}\right.$ vs. $(24.5 \pm 3.6) \mathrm{kg} /$ $\left.\mathrm{m}^{2}, p=0.014\right]$. The proportion of leukocytes $>10 \times 10^{9} / \mathrm{L}$ (71.4\% vs. $15.3 \%, p<0.001)$, lymphocytes $<0.8 \times 10^{9} / \mathrm{L}$ ( $71.4 \%$ vs. $43.3 \%, p=0.011)$, albumin $<35 \mathrm{~g} / \mathrm{L}(28.6 \%$ vs. $8.4 \%, p=0.006), \mathrm{PO}_{2} / \mathrm{FiO}_{2}<300 \mathrm{mmHg}(76.2 \%$ vs $52.4 \%$, $p=0.032)$ and radiologic cavities ( $14.3 \%$ vs. $2.4 \%, p=$ $0.009)$, were significantly higher in the IPA patients; while, serum procalcitonin $(\mathrm{PCT})>0.1 \mathrm{ng} / \mathrm{ml}(9.5 \%$ vs. $47.3 \%, p=0.001$ ) was more common in the control patients. More IPA patients used corticosteroids inhalers $(14.3 \%$ vs. $0.0 \%, p<0.001)$ and systemic corticosteroids ( $85.7 \%$ vs. $17.0 \%, p<0.001)$ before IPA diagnosis. However, no significant differences in the dose of systemic corticosteroids was observed.

Complications of respiratory failure $(100.0 \%$ vs. $21.7 \%, p<0.001)$, heart failure $(42.9 \%$ vs. $20.5 \%, p=$ $0.028)$ and septic shock ( $85.7 \%$ vs. $2.7 \%, p<0.001)$ were more frequent in IPA patients. The proportion of patients needing noninvasive ventilation $(42.9 \%$ vs. $22.3 \%, p=0.014)$, invasive ventilation $(85.7 \%$ vs. $20.8 \%, p<0.001)$ and vasopressor use $(52.4 \%$ vs. $2.4 \%, p<0.001)$, were higher in IPA patients. More IPA patients were admitted to ICU $(71.4 \%$ vs. $24.9 \%$, $p<0.001)$ and had a higher 60 -day mortality rate $(42.9 \%$ vs. $18.9 \%, p=0.015)$ (Table 2$)$.

\section{Diagnosis of IPA}

The mean duration from the diagnosis of IPA to the day of admission was $6.4 \pm 4.8$ days, with a range of $2-18$ days. A serum GM test was performed in 15 of the 21 IPA patients. Seventeen IPA patients were subjected to a GM test in BAL. Fourteen patients had a GM optical index on BAL of $\geq 1.0$, five patients a GM optical index of single serum of $\geq 0.5$.

In all 21 IPA patients, a BAL culture was performed that led to the identification of Aspergillus in 6 patients' cultures. Two patients were diagnosed as proven IPA by trans-bronchial lung biopsy (both were Aspergillus fumigatus). A probable IPA diagnosis was performed in 19 of the 21 IPA patients (Table 3). 
Table 1 Demographic and clinical characteristics between the two groups

\begin{tabular}{|c|c|c|c|c|}
\hline Variables & Total $(\boldsymbol{n}=693)$ & IPA group $(\boldsymbol{n}=21)$ & Control group $(\boldsymbol{n}=672)$ & $p$-value ${ }^{a}$ \\
\hline Age (years, median, IQR) & $61.0(36.0-76.0)$ & $67.0(61.0-82.0)$ & $60.0(36.0-76.0)$ & $<0.001$ \\
\hline Male $(n, \%)$ & $461(66.5)$ & $18(85.7)$ & $443(65.9)$ & 0.058 \\
\hline $\mathrm{BMI}\left(\mathrm{kg} / \mathrm{m}^{2}\right.$, mean $\left.\pm \mathrm{SD}\right)$ & $24.4 \pm 3.6$ & $21.5 \pm 0.4$ & $24.5 \pm 3.6$ & 0.014 \\
\hline Comorbidities $(n, \%)$ & $402(58.0)$ & $18(85.7)$ & $384(57.1)$ & 0.009 \\
\hline Diabetes mellitus & $92(13.3)$ & $15(71.4)$ & $77(11.5)$ & $<0.001$ \\
\hline Cerebrovascular disease & $72(10.4)$ & $4(19.0)$ & $68(10.1)$ & 0.338 \\
\hline COPD & $40(5.8)$ & $6(28.6)$ & $34(5.1)$ & $<0.001$ \\
\hline Asthma & $19(2.7)$ & $3(14.3)$ & $16(2.4)$ & 0.009 \\
\hline Chronic kidney disease & $16(2.3)$ & $2(9.5)$ & $14(2.1)$ & 0.134 \\
\hline Malignant solid tumor & $16(2.3)$ & $0(0.0)$ & $16(2.4)$ & $>0.999$ \\
\hline Chronic congestive heart failure & $3(0.4)$ & $3(14.3)$ & $0(0.0)$ & $<0.001$ \\
\hline Smoking history $(n, \%)$ & $243(35.1)$ & $7(33.3)$ & $236(35.1)$ & 0.866 \\
\hline Alcoholism history ( $n, \%)$ & $92(13.3)$ & $0(0.0)$ & $92(13.7)$ & 0.135 \\
\hline Antibiotics use before admission ( $n, \%)$ & $587(84.7)$ & $15(71.4)$ & $572(85.1)$ & 0.159 \\
\hline \multicolumn{5}{|l|}{ Clinical characteristics $(n, \%)$} \\
\hline Confusion & $32(4.6)$ & $0(0.0)$ & $32(4.8)$ & 0.620 \\
\hline $\mathrm{SBP}<90 \mathrm{mmHg}$ & $8(1.2)$ & $1(4.8)$ & $7(1.0)$ & 0.593 \\
\hline Leukocytes $>10 \times 10^{9} / \mathrm{L}$ & $118(17.0)$ & $15(71.4)$ & $103(15.3)$ & $<0.001$ \\
\hline Lymphocytes $<0.8 \times 10^{9} / \mathrm{L}$ & 299/677 (44.2) & $15(71.4)$ & 284/656 (43.3) & 0.011 \\
\hline $\mathrm{HB}<100 \mathrm{~g} / \mathrm{L}$ & $69(10.0)$ & $5(23.8)$ & $64(9.5)$ & 0.075 \\
\hline Albumin $<35 \mathrm{~g} / \mathrm{L}$ & $58 / 639(9.1)$ & $6(28.6)$ & $52 / 618(8.4)$ & 0.006 \\
\hline $\mathrm{BG}>11 \mathrm{mmol} / \mathrm{L}$ & $48(6.9)$ & $0(0.0)$ & $48(7.1)$ & 0.405 \\
\hline $\mathrm{BUN}>7 \mathrm{mmol} / \mathrm{L}$ & $183 / 685(26.7)$ & $9(42.9)$ & $174 / 664(26.2)$ & 0.090 \\
\hline Serum PCT $>0.1 \mathrm{ng} / \mathrm{ml}$ & 248/541 (45.8) & $2(9.5)$ & $246 / 520(47.3)$ & 0.001 \\
\hline $\mathrm{PO}_{2} / \mathrm{FiO}_{2}<300 \mathrm{mmHg}$ & $340 / 639(53.2)$ & $16(76.2)$ & $324 / 618(52.4)$ & 0.032 \\
\hline \multicolumn{5}{|l|}{ Radiology $(n, \%)$} \\
\hline Cavity & $19(2.7)$ & $3(14.3)$ & $16(2.4)$ & 0.009 \\
\hline Multiple nodules & $151(21.8)$ & $8(38.1)$ & $143(21.3)$ & 0.116 \\
\hline ICs use before IPA diagnosis ( $n, \%)$ & $3(0.4)$ & $3(14.3)$ & $0(0.0)$ & $<0.001$ \\
\hline Systemic corticosteroids use before IPA diagnosis $(n, \%)$ & $132(19.0)$ & $18(85.7)$ & $114(17.0)$ & $<0.001$ \\
\hline Dose of systemic corticosteroids ${ }^{a}$ (mg/kg, mean \pm SD) & $0.6 \pm 0.3$ & $0.6 \pm 0.3$ & $0.6 \pm 0.3$ & 0.853 \\
\hline Early NAls ${ }^{b}$ use $(n, \%)$ & $232(33.3)$ & $10(47.6)$ & $222(33.0)$ & 0.163 \\
\hline Coinfection with other community-acquired pathogens $(n, \%)$ & $265(38.2)$ & $3(14.3)$ & $262(39.0)$ & 0.016 \\
\hline
\end{tabular}

$I Q R$ interquartile range, $S D$ standard deviation, $B M I$ body mass index, COPD chronic obstructive pulmonary disease, $S B P$ systolic blood pressure, $R R$ respiratory rates, $W B C$ white blood cell count, $H B$ hemoglobin, $B G$ blood glucose, $B U N$ blood urea nitrogen, $P C T$ procalcitonin, $P O_{2} / F i O_{2}$ arterial pressure of oxygen/fraction of inspiration oxygen, ICs inhaled corticosteroids, NAIs neuraminidase inhibitors; a: methylprednisolone or its equivalent; b: Neuraminidase inhibitors refer to any dose of oseltamivir, zanamivir, and peramivir; ${ }^{a}$ : IPA group vs control group. The bolded values are $\mathrm{p}$-values $<0.05$, which represent significant differences between subgroups

\section{Coinfection with non-Aspergillus pathogens isolated in FluA-p patients}

Coinfection with other community-acquired pathogens was diagnosed in only 3 of 21 IPA patients and 1 patient was diagnosed with $S$. pneumoniae, 1 patient with $P$. aeruginosa and 1 with K. pneumoniae. While, 39.0\% (262/ 672) of control patients were diagnosed with non-Aspergillus etiologies, S. pneumoniae was the most common diagnosed pathogen with $33.2 \%$ (87/262), followed by $K$. pneumoniae 30.5\% (80/262) and Staphylococcus aureus 20.6\% (54/262) (Additional file 1: Appendix file 4).

Effect of IPA on the 60-day mortality of FluA-p patients Adjusted for age, gender, comorbidities, blood leukocyte counts $>10 \times 10^{9} / \mathrm{L}$, serum PCT $>0.1 \mathrm{ng} / \mathrm{ml}$, coinfection with other pathogens and early NAIs use, a Cox proportional hazard model showed that IPA was associated with an increased risk in the 60-day mortality of FluA-p 


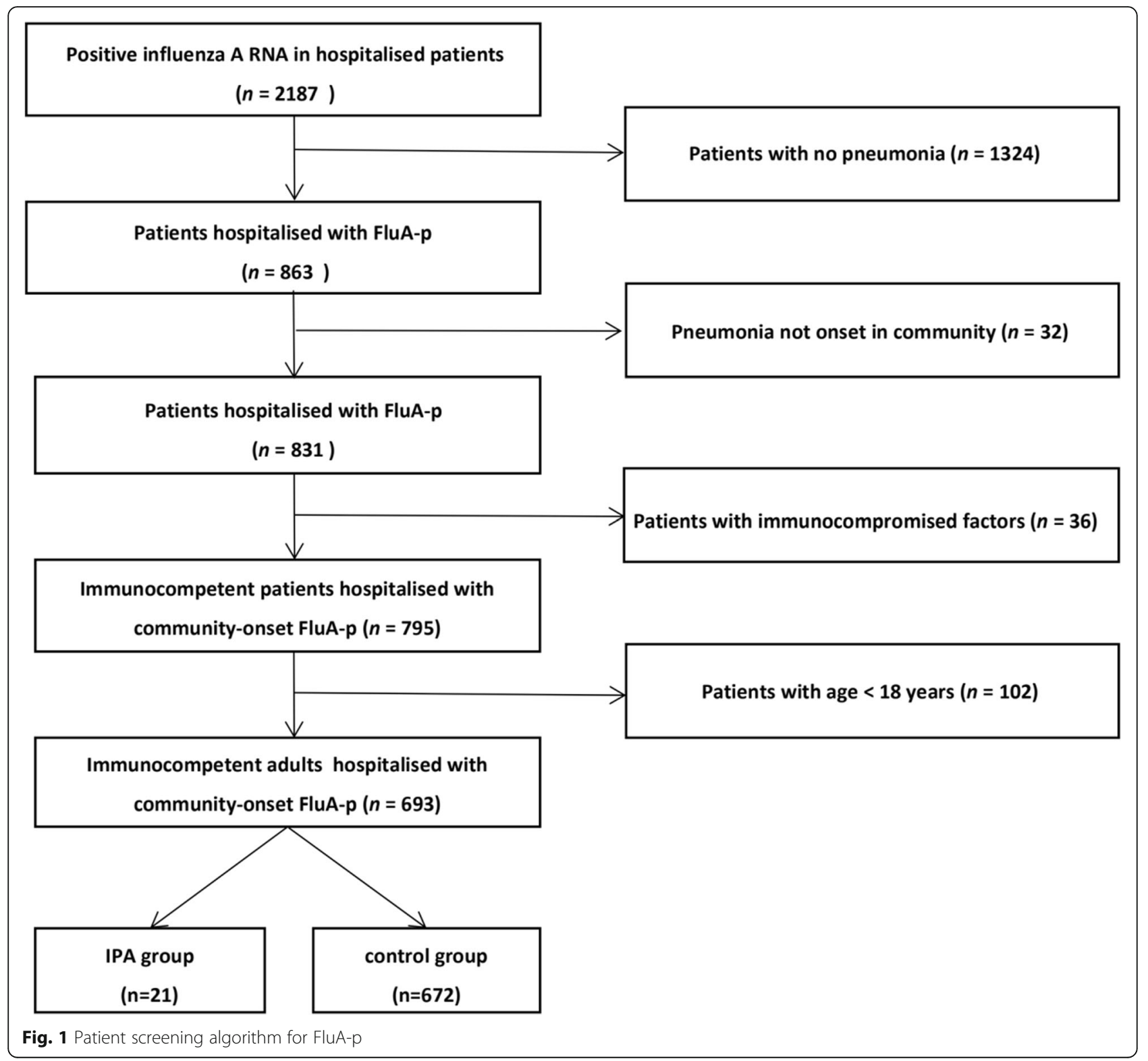

patients [hazard ratio $(H R)$ 4.336, 95\% confidence interval (CI) 1.191-15.784, $p=0.026$ ) (Table 4).

Kaplan-Meier survival curve showed that the 60-day mortality of the IPA patients was significantly higher than that of the control patients $(p=0.006$ for the log rank test) (Fig. 2).

\section{Risk factors associated with IPA occurrence in FluA-p patients}

To explore the risk factors for IPA acquisition, the following variables were entered into the backstep logistic regression model: age, BMI, diabetes mellitus, asthma, chronic congestive heart failure, leukocytes $>10 \times 10^{9} / \mathrm{L}$, lymphocytes $<0.8 \times 10^{9} / \mathrm{L}$, albumin $<35 \mathrm{~g} / \mathrm{L}$, serum PCT $>0.1 \mathrm{ng} / \mathrm{ml}$, cavity on chest radiology, use of inhaled corticosteroids and systemic corticosteroids before IPA diagnosis, and coinfection with other community-acquired pathogens, and the analyses led to the following results: age (OR 1.147, 95\% CI 1.048$1.225, p=0.003)$, systemic corticosteroids use before IPA diagnosis (OR 33.773, 95\% CI 5.681-76.764, $p<0.001$ ), leukocytes > $10 \times 10^{9} / \mathrm{L}($ OR 1.988 , 95\% CI 1.028-6.454, $p=0.029)$ and lymphocytes $<0.8 \times 10^{9} / \mathrm{L}$ on admission (OR 34.813, 95\% CI 1.676-73.006, $p=0.022$ ). These results were proven to be independently related to the IPA acquisition in FluA-p patients (Table 5).

Predictors for a 60-day mortality of IPA in FluA-p patients The demographic features and comorbidities were similar between the survival and deceased patients with IPA. 
Table 2 Supportive treatments and clinical outcomes between the two groups

\begin{tabular}{|c|c|c|c|c|}
\hline Variables & Total $(\boldsymbol{n}=693)$ & IPA group $(\boldsymbol{n}=21)$ & Control group $(\boldsymbol{n}=672)$ & $p$-value ${ }^{\mathbf{a}}$ \\
\hline Vasopressor use $(n, \%)$ & $27(3.9)$ & $11(52.4)$ & $16(2.4)$ & $<0.001$ \\
\hline Length of vasopressor use (days, median, IQR) & $1.0(0.5-3.0)$ & $2.0(0.5-4.5)$ & $1.5(1.0-2.0)$ & 0.185 \\
\hline Noninvasive ventilation $(n, \%)$ & $159(22.9)$ & $9(42.9)$ & $150(22.3)$ & 0.014 \\
\hline Length of noninvasive ventilation (days, median, IQR) & $4.0(1.0-8.0)$ & $2.0(2.0-10.0)$ & $5.0(1.0-8.0)$ & 0.009 \\
\hline Invasive ventilation $(n, \%)$ & $158(22.8)$ & $18(85.7)$ & $140(20.8)$ & $<0.001$ \\
\hline Length of invasive ventilation (days, median, IQR) & $4.0(1.0-9.0)$ & $9.0(7.0-11.0)$ & $4.0(1.0-9.0)$ & 0.003 \\
\hline \multicolumn{5}{|l|}{ Complications during hospitalisation $(n, \%)$} \\
\hline Respiratory failure & $167(24.1)$ & $21(100.0)$ & $146(21.7)$ & $<0.001$ \\
\hline Heart failure & $147(21.2)$ & $9(42.9)$ & $138(20.5)$ & 0.028 \\
\hline Septic shock & $36(5.2)$ & $18(85.7)$ & $18(2.7)$ & $<0.001$ \\
\hline Acute kidney failure & $27(3.9)$ & $3(14.3)$ & $24(3.6)$ & 0.054 \\
\hline Bloodstream infection & $8(1.2)$ & $0(0.0)$ & $8(1.2)$ & $>0.999$ \\
\hline Admittance to ICU $(n, \%)$ & $176(26.3)$ & $15(71.4)$ & $161(24.0)$ & $<0.001$ \\
\hline Length of stay in ICU (days, median, IQR) & $8.0(6.0-13.0)$ & $9.0(7.0-11.0)$ & $8.0(6.0-13.0)$ & 0.473 \\
\hline LOS (days, median, IQR) & $10.0(8.0-14.0)$ & $24.0(11.0-42.0)$ & $10.0(7.0-13.0)$ & $<0.001$ \\
\hline 60-day mortality $(n, \%)$ & $136(19.6)$ & $9(42.9)$ & $127(18.9)$ & 0.015 \\
\hline
\end{tabular}

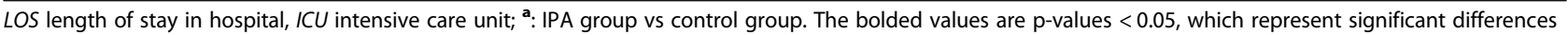
between subgroups

Of the 21 patients that received an antifungal treatment within $24 \mathrm{~h}$ after IPA diagnosis, 18 patients used voriconazole and 3 patients used a combination treatment (voriconazole + echinocandin). However, no significant difference was found in antifungal therapy between the two groups. Compared with the survival group, the deceased patients' group had a higher proportion of lymphocytes $<0.8 \times 10^{9} / \mathrm{L}(100.0 \%$ vs $50.0 \%, p=0.043)$ and lower proportion of early NAIs use $(11.1 \%$ vs $75.0 \%, p=$ 0.014) (Additional file 1: Appendix file 5).

A multivariate logistic regression model confirmed early NAIs use (OR $0.290,95 \%$ CI $0.002-0.584, p=$ 0.021 ) and that was the only predictor for the 60-day mortality in IPA patients (Table 5).

\section{Discussion}

Our study has two important findings: 1) the prevalence of IPA in immunocompetent adult patients hospitalised with FluA-p, was 3.0\%. However, it was associated with increased mortality; 2) we identified age, leukocytes, lymphocytes and systemic corticosteroids use as risk factors for IPA diagnosis. Early NAIs use was related to better outcomes, which were helpful in the prevention and treatment of IPA patients with severe influenza.

There are rare data on the incidence of IPA among all hospitalised FluA-p patients and previous studies were limited to patients admitted to ICU. In our study, the incidence of IPA in ICU patients was $8.2 \%$, which was consistent with the $7.2-8.8 \%$ reported by Rice [20] and

Table 3 Diagnosis of IPA

\begin{tabular}{lll}
\hline Variables & IPA group $(\boldsymbol{n}=21)$ & Control group $(\boldsymbol{n}=672)$ \\
\hline Serum GM test $(n, \%)$ & $15(71.4)$ & $322(47.9)$ \\
BAL GM test $(n, \%)$ & $17(81.0)$ & $167(24.9)$ \\
$\quad$ Serum GM $\geq 0.5$ & $5(4.8)$ & $0(0.0)$ \\
BAL GM $\geq 1.0$ & $14(66.7)$ & $0(0.0)$ \\
BAL Aspergillus culture $(n, \%)$ & $21(100.0)$ & $146(21.7)$ \\
$\quad$ Positive & $6(28.6)$ & $0(0.0)$ \\
Lung tissue microscopy $(n, \%)$ & $4(19.0)$ & $18(2.7)$ \\
$\quad$ Positive & $2(9.5)$ & $0(0.0)$ \\
Proven IPA $(n, \%)$ & $2(9.5)$ & $0(0.0)$ \\
Probable IPA $(n, \%)$ & $19(90.5)$ & $0(0.0)$
\end{tabular}

GM galactomannan, $B A L$ bronchoalveolar lavage 
Table 4 The impact of IPA on the 60-day mortality in FluA-p patients

\begin{tabular}{|c|c|c|c|c|}
\hline \multirow[t]{2}{*}{ Variable } & \multicolumn{2}{|c|}{ Univariate Cox regression } & \multicolumn{2}{|c|}{ Multivariate Cox regression } \\
\hline & $H R(95 \% \mathrm{Cl})$ & $\overline{p \text {-value }}$ & a adjusted $\boldsymbol{H R}(95 \% \mathrm{Cl})$ & $\overline{p \text {-value }}$ \\
\hline$\overline{\mathrm{IPA}}$ & $3.219(1.328-7.803)$ & 0.010 & $4.336(1.191-15.784)$ & 0.026 \\
\hline
\end{tabular}

$H R$ hazard ratio, $\mathrm{Cl}$ interval confidence

a adjusted by age, gender, comorbidities(chronic pulmonary disease, cerebrovascular disease, asthma, diabetes mellitus, chronic kidney disease, malignant solid tumor, chronic congestive heart failure), leukocytes $>10 \times 10^{9}$ / $\mathrm{L}$, serum procalcitonin $>0.1 \mathrm{ng} / \mathrm{ml}$, coinfection with non-Aspergillus other pathogens, early NAls use

Martin-Loeches [21]; but, this was lower than the reported $19.2 \%$ in the Schauwvlieghe's study [22]. Further analysis revealed that all IPA patients had respiratory failure complications and an IPA incidence of $12.6 \%$. This observation is very close to that of the Schauwvlieghe's study, in which the IPA incidence was $14.2 \%$ in non-immunocompromised severe influenza patients. The difference in reported IPA incidences could be explained by the severity of influenza illness, the detection capability of IPA and the discrepancy in ICU admission criteria in the different medical systems.

Although the IPA incidence in our study was not high, the 60 -day mortality was as high as $42.9 \%$, which was in accordance with previous reports of $33-71 \%[12,14-16$, 19, 22-24]. In addition to the genetic background, there were at least two reasons for the difference in mortality among those studies: 1) the influence of the patients' immunity status before influenza onset. In the Schauwvlieghe's study [22], the 90-day mortality in immunocompetent patients with IPA, was 33\%; while, it was $71 \%$ in patients with immunocompromised factors. Among Huang's research population [24], 24\% received immunosuppressive agents and the overall ICU mortality was $41.3 \%$. All the patients in our study were previously immunocompetent and the mortality was lower than that in the previously reported immunocompromised patients' studies. 2) the outcomes were profoundly affected by the awareness, timing and approach of pathogenic testing that were performed for invasive aspergillosis, leading to a delayed IPA diagnosis and treatment. For example, a center in the Schauwvlieghe's [22] study paid more attention to IPA because of several previous reports. As a result, the diagnostic and survival rate of IPA were much higher than that in other centers. Adjusted by age, sex, comorbidities, early use of NAIs and probable co-infection with other etiologies, a Cox proportional hazard model showed, that IPA independently increases the risk for 60-day mortality in FluA-p patients, by 3 times. Our results implied the importance of IPA screening in patients with severe influenza pneumonia, regardless of their previous immunity status. This allows an early diagnosis in patients, that prompts carrying out an antifungal treatment, as soon as possible.

In our study, IPA patients were older in age and with frequent comorbidities. Diabetes and chronic airway diseases (such as COPD and asthma) were the most common underlying diseases. Hypoproteinemia was common, suggesting that their nutritional statuses were poor. When the radiological findings of IPA patients were reviewed, it showed mainly pulmonary infiltrates, with a slightly higher proportion of cavities than the control patients; but, no obvious halo sign was found. Previous studies also showed that the halo sign was only seen in less than $5 \%$ of patients with secondary IPA influenza $[25,26]$. The multivariate logistic regression analysis confirmed, that age, increased leukocyte counts, decreased lymphocyte counts and systemic corticosteroids use, were independent risk factors for the acquisition of IPA in immunocompetent patients hospitalised with FluA-p. It is believed that the pathogenesis of invasive aspergillosis, in the setting of influenza infection, may be due to both local and systemic effects of the virus. Local effects include influenza and inflammation damage of the bronchoalveolar epithelial cells, that lead to the impairment of the barrier function and dysfunction of ciliary motility and clearance [27]. Systemically, influenza alters the Th1/Th2 balance and causes lymphopenia. The immune function of the elderly patients significantly decreased, and their resistance to infection was poor. Previous studies also showed that elderly influenza patients, were more vulnerable to secondary infections [28]. Elevated leukocyte counts in influenza pneumonia patients is usually associated with bacterial or fungal coinfection; while, serum PCT is a relatively specific biomarker of bacterial infection [29]. In our

Table 5 Predictors for acquisition and 60-day mortality of IPA in FluA-p patients

\begin{tabular}{lll}
\hline Predictors for IPA acquisition & p-value & OR (95\% Cl) \\
\hline Age & 0.003 & $1.147(1.048-1.225)$ \\
Systemic corticosteroids use before IPA diagnosis & $<0.001$ & $33.773(5.681-76.764)$ \\
Leukocytes $>10 \times 10^{9} / \mathrm{L}$ & 0.029 & $1.988(1.028-6.454)$ \\
Lymphocyts $<0.8 \times 10^{9} / \mathrm{L}$ & 0.022 & $34.813(1.676-73.006)$
\end{tabular}

\section{Predictors for 60-day mortality of IPA patients}




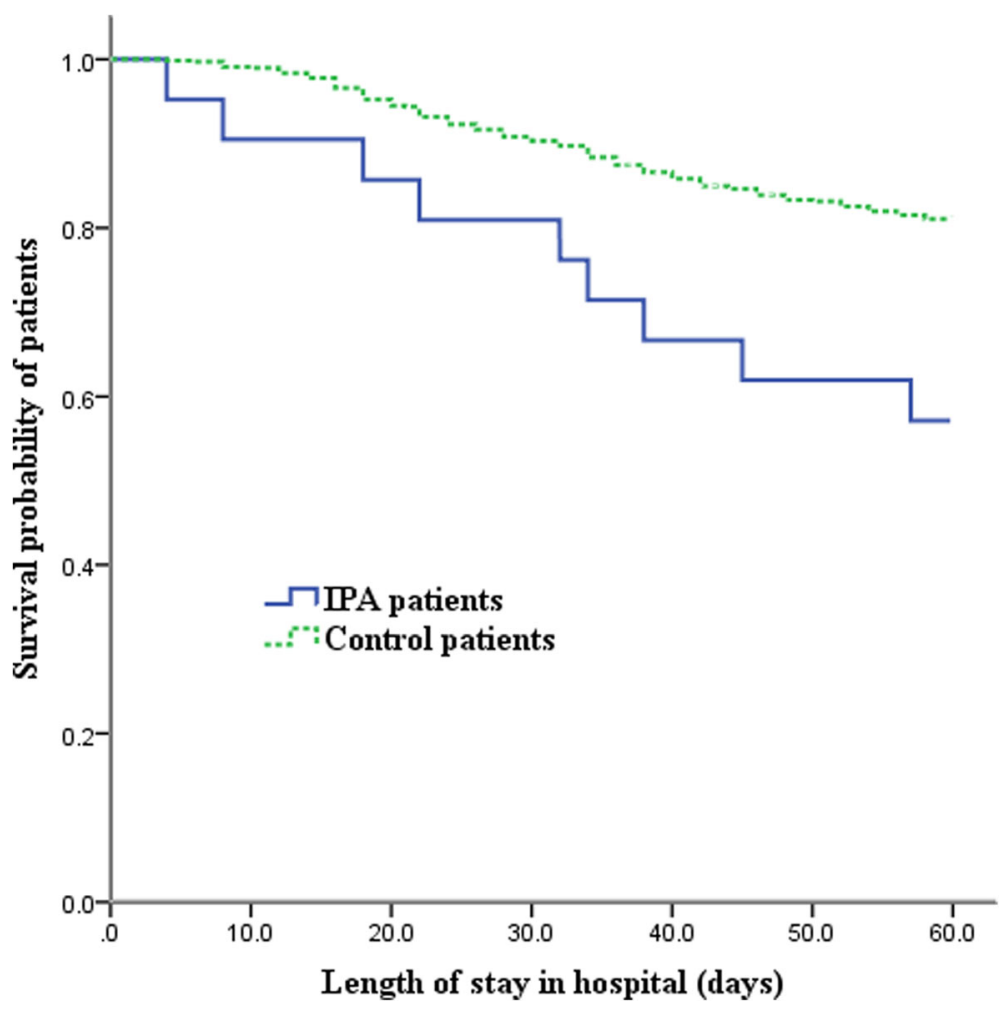

Fig. 2 Kaplan-Meier survival graph for FluA-p patients with and without IPA (censored at 60d)

study, more than $90 \%$ of IPA patients had a serum PCT of $<0.1 \mathrm{ng} / \mathrm{ml}$, indicating that the coinfection with bacteria was not common. Therefore, the elevated leukocytes with normal serum PCT level, strongly suggested fungal infection. Lymphocytes reflected the function of cellar immunity, which was the main anti-viral mechanism in humans. The suppression of cellar immunity delays the clearance of the virus, along with a more serious damage of airway, thus creating conditions for an invasive Aspergillus infection.

The steroids are the most common immunomodulators in clinic, with powerful depressive effects on both cellar immunity and humoral immunity. Corticosteroids use often causes secondary fungal infection [30]. Our study confirmed the association of systemic corticosteroids use and occurrence of IPA as previously reported $[22,24]$. Meanwhile, we found, that even a low-tomoderate dose and short-term systemic corticosteroids use, would increase the risk for of IPA acquisition in the predisposition to immune suppression, caused by severe influenza. Traditionally, it was believed that a prolonged use of steroids (at least 3 weeks and a prednisone equivalent of $>0.3 \mathrm{mg} / \mathrm{kg} / \mathrm{d}$ ) was related to IPA occurrence [31]. It should be noted that there may be overuse of systemic steroids in influenza patients. In numerous reports, more than $50 \%$ of influenza patients received systemic corticosteroids, which were proven to be associated with poor outcomes [32, 33]. In our study, $19 \%$ of FluA-p patients received systemic steroids during hospitalisation. Although in severe influenza, uncontrolled immune response is involved in organic damage and increased mortality. In animal models, corticosteroid treatment was found to decrease mortality and ameliorate acute lung injury induced by influenza [34]. By now, except for septic shock patients with vasopressordependence or adrenal insufficiency, there is no consensus on steroids use in the treatment of severe influenza. Our study confirmed, that the systemic steroids use, was associated with increased risk for IPA and with an unacceptable high mortality, and even in prior immunocompetent FluA-p patients. Therefore, we thought that it was urgent to regulate the use of systemic corticosteroids in the influenza setting.

In our study, early NAIs use was proven to be the only predictor associated with better outcomes in IAPA patients, by decreasing the risk of more than $70 \%$ for a 60 day mortality. Previous studies also proved that the sooner NAIs were used, the better were the outcomes in severe influenza patients $[35,36]$. According to the mechanism of IAPA, it is reasonable that early inhibition of viral duplication and alleviation of lung damage, caused by virus and inflammation, can create favorable conditions for controlling the occurrence and development of IAPA. However, why early use of NAIs had not 
shown a decrease in the risk for IAPA incidence, was still unclear. Apart from the small size of the population, our study implies that there is a complex pathogenesis for IPA occurrence in severe influenza patients.

As far as we know, this was the only investigation focused on IPA in immunocompetent patients hospitalised with FluA-p. Unlike other studies that were limited to ICU patients, our study included, not only patients in ICU, but also patients from general wards. IPA patients, that were not admitted to ICU in other studies and for some reasons, could be included into our population; which, reduced the selective bias. In addition, the population in our study had no classic immunocompromised factors. The occurrence of IPA in these patients was most likely to be neglected. Therefore, the results of our study provide a great significance for clinicians as it allows to identify patients with increased risk for IPA acquisition and at an early stage, advocating therefore, a prompt prevention and treatment.

There were some limitations specific to our study: 1) Besides the nature of the retrospective study, the relatively small sample size along with some missing data, might limit the accuracy of the results; 2) Though the latest diagnostic criteria of IPA was used, the proportion of the microbiologic examinations for IPA in the control patients, was low. Especially, BAL samples were only performed in less than 25\%; while, a serum GM testing was performed in $47 \%$ of control patients. Some studies showed, that with the current diagnostic standard of serum GM optical index $(\mathrm{OD})>0.5$, nearly half of IPA cases would be under-detected [37, 38]. In addition, the triggers to perform BAL were not clarified due to the restrospective study design, and IPA complicating influenza might develope post-admission. This may have limited the number of true cases found and caused selective bias. Therefore, the actual IPA incidence in our study might be under-detected; 3 ) more than $1 / 3$ of the patients had not performed influenza subtype testing and other respiratory tract viruses were not routinely detected. Thus, we could not exclude coinfection with other viruses.

\section{Conclusions}

Our study showed that there is approximatively 3.0\% of IPA incidence with an increased mortality that was observed even in immunocompetent patients, hospitalised with FluA-p. Additionally, we identified age, elevated leukocytes, reduced lymphocytes, on the day of admission and systemic corticosteroids use, as risk factors for IPA acquisition, and that early NAIs use was a predictor of better outcome. Meanwhile, it is suggested that these results should be confirmed using prospective and large sample studies to further verify these conclusions.

\section{Supplementary information}

Supplementary information accompanies this paper at https://doi.org/10. 1186/s12890-020-01257-W.

Additional file 1 : Appendix 1: Details of Participating centers. Appendix 2 Definition of microbiological criteria of coinfected other pathogens. Appendix 3 Definition of underlying diseases. Appendix 4 Coinfection with other pathogens. Appendix $\mathbf{5}$ Univariate analysis between the survival group and the deceased group.

\section{Abbreviations}

Flu-p: Influenza-related pneumonia; IPA: Invasive pulmonary aspergillosis; NAl: Neuraminidase inhibitor; OR: Odds ratio; HR: Hazard ratio; 95\% IC: 95\% Interval confidence; FluA-p: Influenza A-related pneumonia; RT-PCR: Reverse transcription polymerase chain reaction; EORTC/MSG: European Organization for Research and Treatment of Cancer/Invasive Fungal Infections Cooperative Group and the National Institute of Allergy and Infectious Diseases Mycoses Study Group; BAL: Bronchoalveolar lavage; GM: Galactomannan; IQR: Interquartile range; BMI: Body mass index; COPD: Chronic obstructive pulmonary disease; SBP: Systolic blood pressure; Hb: Hemoglobin; BG: Blood glucose; ALB: Albumin; BUN: Blood urea nitrogen; PH: Hydrogen ion index; $\mathrm{pO}_{2} / \mathrm{FiO}_{2}$ : Arterial pressure of oxygen/fraction of inspiration oxygen; ICs: Inhaled corticosteroid; PCT: Procalcitonin; ICU: Intensive care unit

\section{Acknowledgments}

We thank Dr. Bai and Dr. Zhang (Infectious Disease Department, Beijing

Jishuitan Hospital) for their valuable comments on the manuscript.

Conflict of interest

None.

Authors' contributions

Study concept and design: $L C, X d H$. Acquisition of data: LC, XdH, YIL, CXZ, XqX. Statistical analysis of data: LC. Drafting of the manuscript: LC. Critical revision of the manuscript for important intellectual content: $\mathrm{XdH}, \mathrm{XqX}$. All authors agree with the article submission. All authors read and approved the final manuscript.

Funding

This study is founded by Beijing JST research (ZR-201921).

Availability of data and materials

All data generated or analysed during this study are included in this published article and its supplementary information files.

\section{Ethics approval and consent to participate}

The study design was approved by the Ethics Committee of Beijing Jishuitan Hospital (No.201911-15). Given the retrospective nature of the study, the Ethics Committee determined that an informed consent was not necessary.

Consent for publication

Not applicable.

\section{Competing interests}

The authors declare that they have no competing interests.

\section{Author details}

${ }^{1}$ Department of Infectious Diseases, Beijing Jishuitan Hospital, 4th Medical College of Peking University, Beijing, China. ${ }^{2}$ Department of Pulmonary and Critical Care Medicine, Qingdao Municipal Hospital, Qingdao City, Shandong Province, China. ${ }^{3}$ Department of Infectious Diseases and Clinical

Microbiology, Beijing Chao-Yang Hospital, Capital Medical University, Beijing, China. ${ }^{4}$ Department of Pulmonary and Critical Care Medicine, Beijing Huimin Hospital, Beijing, China. ${ }^{5}$ Department of Pulmonary and Critical Care Medicine, the 2nd People's Hospital of Yunnan Province, Kunming City, Yunnan Province, China. 
Received: 2 April 2020 Accepted: 5 August 2020

Published online: 09 September 2020

\section{References}

1. Peteranderl C, Herold S, Schmoldt C. Human influenza virus infections. Semin Respir Crit Care Med. 2006;37(4):487-500.

2. Neuzil KM, Bresee JS, de la Hoz F, Johansen K, Karron RA, Krishnan A, et al. Data and product needs for influenza immunization programs in low- and middle-income countries: rationale and main conclusions of the WHO preferred product characteristics for next-generation influenza vaccines. Vaccine. 2017;35(43):5734-7.

3. Dahal S, Jenner M, Dinh L, Mizumoto K, Viboud C, Chowell G. Excess mortality patterns during 1918-1921 influenza pandemic in the state of Arizona, USA. Ann Epidemiol. 2016;28(5):273-80.

4. Kumar V. Influenza in Children. Indian J Pediatr. 2017;84(2):139-43.

5. Lozano R, Naghavi M, Foreman K, Lim S, Shibuya K, Aboyans V, et al. Global and regional mortality from 235 causes of death for 20 age groups in 1990 and 2010: a systematic analfor the global burden of disease study 2010. Lancet. 2012;380(9859):2095-128.

6. Li M, Bolker BM, Dushoff J, Ma J, Earn DJD. Patterns of seasonal and pandemic influenza-associated health care and mortality in Ontario, Canada. BMC Public Health. 2019;19(1):1237.

7. Klein EY, Monteforte B, Gupta A, Jiang W, May L, Hsieh YH, Dugas A. The frequency of influenza and bacterial coinfection: a systematic review and meta-analysis. Influenza Other Respir Viruses. 2016;10(5):394-403.

8. Kylla H, Dutta TK, Roychoudhury P, Subudhi PK. Coinfection of diarrheagenic bacterial and viral pathogens in piglets of northeast region of India. Vet World. 2019;12(2):224-30.

9. Brundage JF. Interactions between influenza and bacterial respiratory pathogens: implications for pandemic preparedness. Lancet Infect Dis. 2016; 6(5):303-12.

10. Harper SA, Bradley JS, Englund JA, File TM, Gravenstein S, Hayden FG, et al. Seasonal influenza in adults and children--diagnosis, treatment, chemoprophylaxis, and institutional outbreak management: clinical practice guidelines of the Infectious Diseases Society of America. Clin Infect Dis. 2019;48(8):1003-32.

11. Cao B, Huang Y, She DY, Cheng QJ, Fan H, Tian XL, et al. Diagnosis and treatment of community-acquired pneumonia in adults: 2016 clinical practice guidelines by the Chinese thoracic society, Chinese Medical Association. Clin Respir J. 2018;12(4):1320-60.

12. Cadena J, Thompson GR 3rd, Patterson TF. Invasive Aspergillosis: current strategies for diagnosis and management. Infect Dis Clin N Am. 2016;30(1): 125-42.

13. Solano C, Vázquez L. Invasive aspergillosis in the patient with oncohematologic disease. Rev Iberoam Micol. 2018;35(4):198-205.

14. Alshabani K, Haq A, Miyakawa R, Palla M, Soubani AO. Invasive pulmonary aspergillosis in patients with influenza infection: report of two cases and systematic review of the literature. Expert Rev Respir Med. 2015;9(1):89-96.

15. Kim SH, Kim MN, Lee SO, Choi SH, Kim YS, Woo JH, et al. Fatal pandemic influenza A/H1N1 infection complicated by probable invasive pulmonary aspergillosis. Mycoses. 2012;55(2):189-92.

16. Lat A, Bhadelia N, Miko B, Furuya EY, Thompson GR 3rd. Invasive aspergillosis after pandemic (H1N1) 2009. Emerg Infect Dis. 2010;16(6):971-3.

17. Chen L, Zhou F, Li H, Xing X, Han X, Wang Y, et al. Disease characteristics and management of hospitalised adolescents and adults with communityacquired pneumonia in China: a retrospective multicentre survey. BMJ Open. 2018;8(2):e018709.

18. Donnelly JP, Chen SC, Kauffman CA, Steinbach WJ, Baddley JW, Verweij PE, et al. Revision and Update of the Consensus Definitions of Invasive Fungal Disease From the European Organization for Research and Treatment of Cancer and the Mycoses Study Group Education and Research Consortium. Clin Infect Dis. 2019;ciz1008.

19. Ku YH, Chan KS, Yang CC, Tan CK, Chuang YC, Yu WL, et al. Higher mortality of severe influenza patients with probable aspergillosis than those with and without other coinfections. J Formos Med Assoc. 2017;116(9):660-70.

20. Rice TW, Rubinson L, Uyeki TM, Vaughn FL, John BB, Miller RR 3rd, et al. Critical illness from 2009 pandemic influenza A virus and bacterial coinfection in the United States. Crit Care Med. 2012;40(5):1487-98.

21. Martin-Loeches I, Schultz JM, Vincent JL, Alvarez-Lerma F, Bos LD, SoléViolán J, et al. Increased incidence of co-infection in critically ill patients with influenza. Intensive Care Med. 2017:43(1):48-58.
22. Schauwvlieghe AFAD, Rijnders BJA, Philips N, Verwijs R, Vanderbeke L, Van Tienen C, et al. Invasive aspergillosis in patients admitted to the intensive care unit with severe influenza: a retrospective cohort study. Lancet Respir Med. 2018;6(10):782-92.

23. Cattaneo C, Gramegna D, Malagola M, Pagani C, Borlenghi E, Cerqui E, et al. Invasive pulmonary aspergillosis in acute leukemia: a still frequent condition with a negative impact on the overall treatment outcome. Leuk Lymphoma. 2019;60(12):3044-50.

24. Huang L, Zhang N, Huang X, Xiong S, Feng Y, Zhang Y, et al. Invasive pulmonary aspergillosis in patients with influenza infection: A retrospective study and review of the literature. Clin Respir J. 2019;13(4):202-11.

25. Vandewoude KH, Blot SI, Depuydt P, Benoit D, Temmerman W, Colardyn F, Vogelaers D. Clinical relevance of Aspergillus isolation from respiratory tract samples in critically ill patients. Crit Care. 2006;10(1):R31.

26. Park SY, Lim C, Lee SO, Choi SH, Kim YS, et al. Computed tomography findings in invasive pulmonary aspergillosis in non-neutropenic transplant recipients and neutropenic patients, and their prognostic value. J Inf Secur. 2011;63(6):447-56.

27. Crum-Cianflone NF. Invasive Aspergillosis Associated With Severe Influenza Infections. Open Forum Infect Dis. 2016;3(3):ofw171.

28. Rosano A, Bella A, Gesualdo F, Acampora A, Pezzotti P, Marchetti S, et al. Investigating the impact of influenza on excess mortality in all ages in Italy during recent seasons (2013/14-2016/17 seasons). Int J Infect Dis. 2019;88: 127-34.

29. Choi JJ, McCarthy MW. Novel applications for serum procalcitonin testing in clinical practice. Expert Rev Mol Diagn. 2018;18(1):27-34.

30. Lionakis MS, Kontoyiannis DP. Glucocorticoids and invasive fungal infections. Lancet. 2003;362(9398):1828-38.

31. Karthaus M, Buchheidt D. Invasive aspergillosis: new insights into disease, diagnostic and treatment. Curr Pharm Des. 2013;19(20):3569-94.

32. Ni YN, Chen G, Sun J, Liang BM, Liang ZA. The effect of corticosteroids on mortality of patients with influenza pneumonia: a systematic review and meta-analysis. Crit Care. 2019;23(1):99.

33. Li H, Yang SG, Gu L, Zhang Y, Yan XX, Liang ZA, et al. Effect of low-tomoderate-dose corticosteroids on mortality of hospitalised adolescents and adults with influenza A(H1N1)pdm09 viral pneumonia. Influenza Other Respir Viruses. 2017;11(4):345-54.

34. Ghoneim HE, McCullers JA. Adjunctive corticosteroid therapy improves lung immunopathology and survival during severe secondary pneumococcal pneumonia in mice. J Infect Dis. 2014;209(9):1459-68.

35. Osthoff M, Erb S. Neuraminidase inhibitors and influenza infection. JAMA Intern Med. 2016;176(3):415.

36. Muthuri SG, Venkatesan S, Myles PR, Leonardi-Bee J, Lim WS, Al Mamun A, et al. Impact of neuraminidase inhibitors on influenza A(H1N1)pdm09related pneumonia: an individual participant data meta-analysis. Influenza Other Respir Viruses. 2016;10(3):192-204.

37. Zhou W, Li H, Zhang Y, Huang M, He Q, Li P, et al. Diagnostic value of Galactomannan antigen test in serum and Bronchoalveolar lavage fluid samples from patients with Nonneutropenic invasive pulmonary Aspergillosis. J Clin Microbiol. 2017;55(7):2153-61.

38. van de Groep K, Verboom DM, van de Veerdonk FL, Haas P-JA, van der Poll $\mathrm{T}$, Schultz MJ, et al. Detection of invasive Aspergillosis in critically ill patients with influenza: the role of plasma Galactomannan. Am J Respir Crit Care Med. 2019;200(5):636-8.

\section{Publisher's Note}

Springer Nature remains neutral with regard to jurisdictional claims in published maps and institutional affiliations. 